\title{
Environmental dependence of SFRs in late-type GAMA galaxies
}

\author{
Meiert W. Grootes ${ }^{1}$, Richard J. Tuffs ${ }^{1}$, Ellen Andrae ${ }^{1}$, \\ Lee S. Kelvin ${ }^{2}$, Jochen Liske ${ }^{3}$, Barry F. Madore ${ }^{4}$, \\ Cristina C. Popescu ${ }^{5}$, Aaron S. G. Robotham ${ }^{2}$, Mark Seibert ${ }^{4}$, \\ Edward N. Taylor ${ }^{6}$, and the GAMA Team
}

\begin{abstract}
${ }^{1}$ Max-Planck-Institut für Kernphysik, Saupfercheckweg 1, 69117 Heidelberg, Germany ;
${ }^{2}$ School of Physics and Astronomy, University of St. Andrews, North Haugh, St. Andrews, KY16 9SS, UK ; ${ }^{3}$ European Southern Observatory, Karl-Schwarzshild Str. 2, 85748, Garching, Germany ; ${ }^{4}$ Observatories of the Carnegie Institution for Science, 813 Santa Barbara Street, Pasadena, CA 91101, USA ; ${ }^{5}$ Jeremiah Horrocks Institute, University of Central Lancashire, Preston PR1 2HE, UK ; ${ }^{6}$ Sydney Institute for Astronomy, School of Physics, University of Sydney, NSW 206, Australia
\end{abstract}

email: meiert.grootes@mpi-hd.mpg.de

\begin{abstract}
Present and past gas-fuelling of galaxies is expected to depend upon both the properties of the galaxies themselves, as well as their larger-scale environments. In the case of galaxies in groups the environment, i.e the group mass, can be probed by measuring the velocity dispersion of the group members, as done with the GAMA Galaxy Group catalogue (Robotham et al. 2011), probing the halo mass function all the way to small groups. The gas-fuelling rate of normal late-type galaxies can be traced by the SFR under the assumption of a steady state between gas-fuelling and gas-consumption by SF. We present a method to estimate disk opacities from UV/optical photometric characteristics, calibrated using the radiative transfer model of Popescu et al. (2011), applied to UV-Opt-FIR GAMA/H-ATLAS photometry for a subset of GAMA galaxies. We use the method to extract attenuation corrected SFRs for a large sample of late-type GAMA galaxies, which we use in an initial application to constrain the dependency of star formation/gas-fuelling in late-type galaxies on mass of parent $\mathrm{DMH}$, and compactness of galaxy group.
\end{abstract}

Keywords. galaxies: fundamental parameters, galaxies: ISM, galaxies: spiral, dust, extinction

\section{Introduction}

While the current observed population of stars in the universe is generally accepted as having formed via condensation out of gas in galaxies, the processes governing the introduction of this gas into the galaxies are not yet fully understood. The two main processes under consideration are i) accretion of gas via mergers of previously gas-rich systems, and ii) cooling and direct accretion of gas from the intergalactic medium (IGM). The latter is arguably the more fundamental of the two, in the sense that it is the process by which the first primordial galaxies presumably formed.

Following the current paradigm of structure formation, baryonic structure follows the hierarchical, gravity-driven formation of dark matter halos(DMH). The propensity for gas to cool and accrete, fuelling existing galaxies or creating new ones, is thus expected to depend upon the galaxy's immediate environment, as determined by the $\mathrm{DMH}$, due to virial heating of the gas in the halo. On the other hand, the efficiency of gas-fuelling is also expected to depend on positive/negative feedback effects linked to the properties of the individual galaxies, thus necessitating an approach capable of separating these 
dependencies. Although considerable theoretical effort has been applied to the subject (Bower et al. 2006), comparison of theoretical predictions with observations has hitherto only been possible for the most massive DMHs $\left(M_{D M H}>10^{14} M_{\odot}\right)$. Using the Galaxy and Mass Assembly survey (GAMA $\dagger$, Driver et al. 2011), and in particular the GAMA Galaxy Group Catalogue $\left(\mathrm{G}^{3} \mathrm{C}\right.$, Robotham et al. 2011) this range can be extended to $\mathrm{DMH}$ with masses on the order of $M_{D M H} \approx 10^{11} M_{\odot}$, probing the mass range of galaxy groups.

Based on the GAMA data, the instantaneous SF rate derived from the NUV can be used as a proxy for the gas-fuelling rate of normal late-type galaxies as appears to be valid for most of these systems (Hopkins \& Beacom 2006), creating a large statistical sample of galaxies in a variety of DMHs ranging from group to cluster masses for which gas-fuelling rates can be obtained.

\section{Late-type galaxies; opacities and SFRs}

The emission of galaxies, especially late-type galaxies, at UV/optical wavelengths is known to be strongly affected by inclination dependent attenuation due to dust concentrated in their disks (Tuffs et al. 2004; Pierini et al. 2004; Driver et al. 2007). As such, any measure of the SFR of galaxies derived from emission in the NUV must be corrected for these effects, in order to obtain an estimate of the sources' intrinsic emission. Estimating the disk opacities of late-type galaxies, allowing attenuation corrections to be applied has, however, proven to be challenging, and various approaches exist. These range from the most powerful using UV-optical-FIR data together with radiative transfer modelling to self-consistently correct for attenuation while taking a source's inclination into account (Popescu et al. 2011), to semi-empirical methods based on UV/optical data alone, e.g the UV-spectral slope $\beta$ (Meurer, Heckmann, \& Calzetti 1999) or the Balmer decrement (Calzetti 2001; Wijesinghe et al. 2011). Regrettably, full coverage of the UV/optical-FIR spectrum, resp. multiple band UV data and spectroscopy, is often not available, making homogeneous approaches on large statistical samples difficult.

Using the GAMA photometry, stellar masses (Taylor et al. 2011), and morphological fits (Kelvin et al. 2011) in combination with the HERSCHEL-ATLAS (H-ATLAS $\ddagger$, Eales et al. 2010) science demonstration phase sources matched to GAMA sources (Smith et al. 2011), we present a correlation between the face-on B-band opacity due to dust $\tau_{B}^{f}$ and the stellar mass surface density $\mu_{*}$ (Grootes et al. 2012a)(cf. Fig. 1)

$$
\log \left(\tau_{B}^{f}\right)=\log \left(\frac{\mu_{*}}{\mathrm{M}_{\odot} \mathrm{kpc}^{-2}}\right) 0.94( \pm 0.06)-8.0( \pm 0.6)
$$

for a sample of late-type sources selected in the NUV-r vs. Sersić index $n$ plane (Driver et al. (2011); Kelvin et al. (2012)), using a separator

$$
(\mathrm{NUV}-r) \leqslant-22.35 \log (n)+11.5
$$

$\dagger$ http://www.gama-survey.org ; GAMA is a joint European- Australasian project based around a spectroscopic campaign using the Anglo-Australian Telescope. The GAMA input catalogue is based on data taken from the Sloan Digital Sky Survey and the UKIRT Infrared Deep Sky Survey. Complementary imaging of the GAMA regions is being obtained by a number of independent survey programs including GALEX MIS, VST KIDS, VISTA VIKING, WISE, Herschel-ATLAS, GMRT and ASKAP providing UV to radio coverage. GAMA is funded by the STFC (UK), the ARC (Australia), the AAO, and the participating institutions.

$\ddagger$ http://www.h-atlas.org ; The Herschel-ATLAS is a project with Herschel, which is an ESA space observatory with science instruments provided by European-led Principal Investigator consortia and with important participation from NASA. 


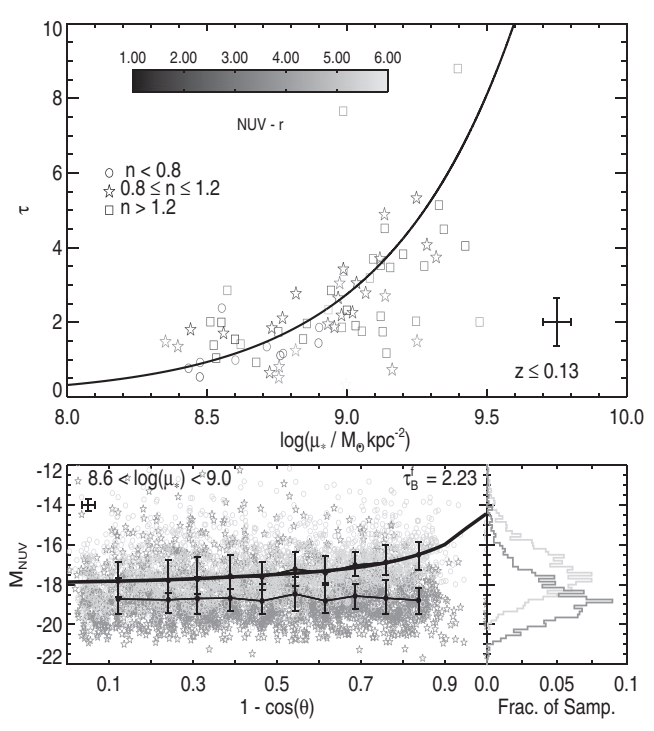

Figure 1. Top: Correlation between $\tau_{B}^{f}$ and $\mu_{*}$. The best fit (Eq.2.1) is shown as dash-dotted line. Bottom: Distribution of $M_{N U V}$ for a complete sample of GAMA late-type galaxies, characterized by $\mu_{*}$ as a function of inclination $\theta$. The bin wise medians in $M_{N U V}$ and $1-\cos (\theta)$ before and after correction are shown by solid lines. The expected uncorrected locus of a fiducial galaxy defined using the model of Popescu et al. (2011) with $\tau_{B}^{f}$ and $M_{N U V}$ corresponding to the medians of the corrected sample is shown as a dash-dotted line. Error bars indicate the interquartile range in each bin.
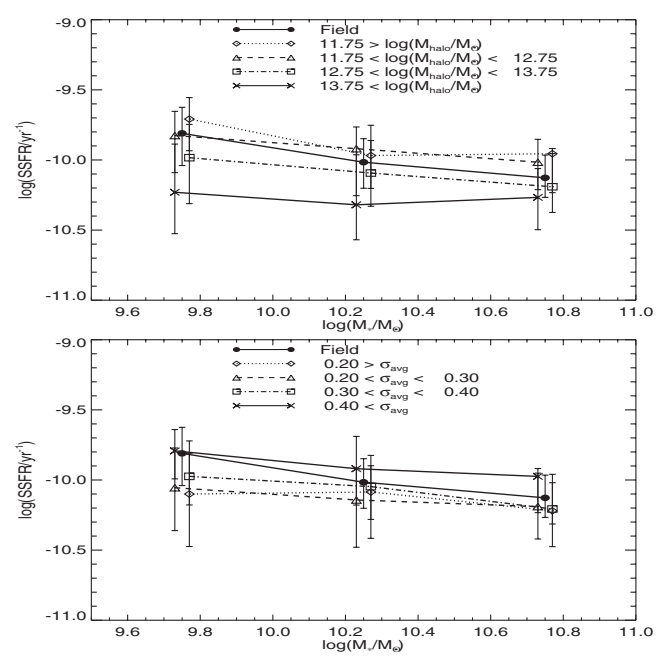

Figure 2. Top: Median SSFR as a function of $M_{*}$ in bins of 0.5 dex for 4 bins of DMH mass ranging from $M_{D M H}<10^{11.75} M_{\odot}$ to $M_{D M H}>10^{13.75} M_{\odot}$ and the comparison field sample. Bottom: Median SSFR as a function $M_{*}$ in bins of 0.5 dex for 4 bins of average link strength $\sigma_{\text {avg }}$ as a probe of local galaxy density and the comparison field sample. Error bars show the interquartile range of the distribution in each bin. The positions of the plotted points have been shifted by \pm 0.02 in $\log \left(M_{*} / \mathrm{M}_{\odot}\right)$ w.r.t. the field sample for visibility.

calibrated on the overlap of GAMA with GALAXY ZOO (Lintott et al. 2008)(see Grootes et al. $2012 \mathrm{~b}$ for details). Eq. 2.1 allows $\tau_{B}^{f}$ to be estimated for statistical samples of late-type galaxies on the basis of optical broad band photometry alone, and is thus homogeneously applicable to large data-sets.

The correlation has been tested using the attenuation-inclination relation for complete samples of late-type galaxies, and successfully corrects the samples for the effects of dust attenuation using the estimated values of $\tau_{B}^{f}$, the relation between $\tau_{B}^{f}$ and intrinsic size for disk galaxies (Möllenhoff, Popescu, \& Tuffs 2006), and the radiative transfer model of Popescu et al. (2011)(cf. Fig. 1). The result has been found to be consistent with, e.g. the mass-metallicity relation and the $M_{g a s} / M_{*}$ vs. $M_{*}$ relation and to be driven largely by a rougly linear relation between dust mass and stellar mass (Grootes et al. 2012a).

Using this correlation we correct the NUV emission of a complete statistical sample of GAMA late-type galaxies with stellar mass $M_{*} \geqslant 10^{9.5} M_{\odot}$ for dust-attenuation and calculate NUV SF rates (SFR) as specified in Wijesinghe et al. (2011).

\section{Environmental dependence of SFRs}

The $\mathrm{G}^{3} \mathrm{C}$ (Robotham et al. 2011) is a catalogue of galaxy groups obtained by applying a friends-of-friends (FoF) algorithm to the GAMA spectroscopic survey which has a 
redshift completeness of $>90 \%$ to its limiting depth of $r_{p e t}<19.4$, providing a novel environmental metric reliably recovering galaxy groups and providing mass estimates of the host DMHs extending down to $M_{D M H} \approx 10^{11} M_{\odot}$. The specific SFR (SSFR) of galaxies is known to depend on galaxy stellar mass so, to disentangle this effect from environmental effects, we compare the SSFR (corrected as specified above) of late-type GAMA galaxies, selected according to Eq. 2.2, as a function of their stellar mass for different DMH mass bins, and a comparison sample of field galaxies, defined as not being grouped in the $\mathrm{G}^{3} \mathrm{C}$ (cf. Fig.2). We find that, while the SSFR of late-type galaxies in halos of $M_{D M H} \leqslant 10^{12.75} M_{\odot}$ is essentially indistinguishable from that of late-type field galaxies at all galaxy stellar masses, the SSFR of galaxies with $M_{*} \leqslant 10^{10.5} M_{\odot}$ in halos with $10^{12.75} M_{\odot}<M_{D M H} \leqslant 10^{13.75} M_{\odot}$ is slightly suppressed w.r.t. the field sample, and strongly suppressed in halos more massive than $10^{13.75} M_{\odot}$. The SSFRs of galaxies with $M_{*}>10^{10.5} M_{\odot}$ do not display any environmental dependence.

The $\mathrm{G}^{3} \mathrm{C}$ also enables the use of grouping parameters as a metric for the group environment. The strength of a link between galaxies in the FoF algorithm can be characterized by the ratio of actual linking length and maximum possible link length, with strongly linked galaxies having small values. Thus, the compactness of a group in redshift space can, to first order, be characterized by the average linking strength $\sigma_{\text {avg }}$ of the group, with small values corresponding to compact groups and larger values to more extended groups. This measure of compactness can be viewed as a proxy for the galaxy density in redshift space, taking only group member galaxies into account. We find that the average SSFR of low mass galaxies is suppressed w.r.t. the field sample in the most compact groups, while the similarity to the field sample increases as the groups become more extended (cf. Fig. 2). Galaxies with $M_{*}>10^{10.5} M_{\odot}$ don't show environmental dependence, and the SSFR suppression of low mass galaxies is less pronounced as a function of group compactness, than as a function of halo mass.

\section{References}

Bower, R. G. \& Benson, A. J., Malbon, R., et al. 2006, MNRAS, 370, 645

Calzetti D. 2001, PASP, 113, 1449

Driver, S. P., Popescu, C. C., Tuffs, R. J., et al. 2007, MNRAS, 379, 1022

Driver, S. P., Hill, D. T., Kelvin, L. S., et al. 2011, MNRAS, 413, 971

Driver, S. P., et al. 2012, in prep.

Eales, S., Dunne, L., Clements, D., et al. 2010, PASP, 122, 499

Grootes, M. W., et al. 2012a, in prep.

Grootes, M. W., et al. 2012b in prep.

Hopkins, A. M. \& Beacom, J. F. 2006, ApJ, 651, 142

Kelvin L. S., Driver, S. P., Robotham, A. S. G., et al. 2011, MNRAS, submitted

Kelvin L. S., et al. 2012, in prep.

Meurer G. R., Heckmann T. M., \& Calzetti D. 1999, ApJ, 521, 64

Möllenhoff C., Popescu C. C. \& Tuffs R. J. 2006, A\&SA, 456, 941

Lintott, C. J., Schawinski, K., Slosar, A., et al. 2008, MNRAS, 389, 1179

Pierini D., Gordon K. D., Witt A. N., \& Madsen G. J. 2004, ApJ, 617, 1022

Popescu, C. C. et al. 2000, A\&̈A, 362, 138

Popescu, C. C., Tuffs, R. J., Dopita, M. A., et al. 2011, A\&SA, 527, A109+

Robotham, A. S. G., Norberg, P., Driver, S. P., et al. 2011 MNRAS,

Smith, D. J. B., Dunne, L., Maddox, S. J., et al. 2011, MNRAS, 416, 857

Taylor, E. N., Hopkins, A. M., Baldry, I. K., et al. 2011, arXiv:1108.0635, accepted by $M N R A S$

Tuffs, R. J., Popescu, C. C., Völk, H. J., Kylafis, N. D., \& Dopita, M. A. 2004, A\&SA, 419, 821

Wijesinghe D., Hopkins, A. M., Sharp, R., et al. 2011, MNRAS, 415, 1002 


\section{Discussion}

MADORE: Could you remind us what the typical value for the face-on extinction is for spiral galaxies corrected by this method?

Grootes: This depends both on the wavelength considered, as well as the stellar mass. Additionally there is a spread in the value of opacity for a given stellar mass. Nevertheless a typical spiral galaxy would have a face-on B-band central optical depth of about 4, for which an attenuation of the integrated emission of $\sim 0.9$ mag in the NUV and $\sim 0.3 \mathrm{mag}$ in the $\mathrm{g}$ band is found for a median inclination of 60 degrees.

HolWERDA: How sensitive are you to different scale lengths for dust in the conversion from $\mu_{*}$ to $\tau_{B}^{f}$ ?

Grootes: Converting $\mu_{*}$ to $\tau_{B}^{f}$ uses the relative values of the geometrical components of the model to be a correct representation of the galaxy being treated. The model is calibrated on a sample of well-resolved edge-on galaxies with intermediate morphologies along the Hubble sequence of late-type galaxies (see Popescu et al. 2000). For these objects the relative scale lengths of the dust and stars is directly determinable through radiation transfer-modelling of the images, and is found to be rather constant. In moving to earlier or later-type disk galaxies there is some degree of flexibility incorporated via the bulge-to-disk ratio, which takes care of the general trend for the overall distribution of older stars in the galaxy (the combined population of old stars in the bulge and disk) to be more centrally concentrated compared to the dust disk when moving towards earlier types on the Hubble sequence. Nevertheless, we expect that our model would start to fail at some level if the ratio of scale lengths of dust in the disk and stars in the disk would markedly change for very early-type or very late type spiral galaxies. As discussed at length in Popescu et al. (2011) any such failure would however be recognisable through a shift in the relation between the observed and predicted colour-surface brightness relation of the dust emission of spiral galaxies. 